\title{
Caracterización genética de cepas de Listeria monocytogenes aisladas durante los años 2007-2014 en Chile
}

\author{
Soledad Ulloa', Loredana Arata ${ }^{1}$, Pedro Alarcón², Pamela Araya², \\ Juan Carlos Hormazábal² y Jorge Fernández'
}

\section{Genetic characterization of Listeria monocytogenes strains isolated during 2007-2014 in Chile}

Background: Listeria monocytogenes is a foodborne pathogen that causes listeriosis, a disease that can present as febrile gastroenteritis or as an invasive form that has high mortality rates. So far, the genetic diversity of strains of L. monocytogenes isolated from patients, foods and environmental sources in Chile has been poorly studied. Aim: To characterize genetically L. monocytogenes strains received by the Institute of Public Health of Chile (ISP) between 2007 and 2014. Methods: We selected 94 strains of L. monocytogenes corresponding to 94 different pulsotypes identified by pulsed field gel electrophoresis (PFGE), DNA was extracted and serotyping was performed by polymerase chain reaction (PCR) and multilocus sequence typing (MLST). Results: The most common serotype was $4 \mathrm{~b}(55.3 \%)$, followed by serotypes $1 / 2 \mathrm{a}(25.5 \%), 1 / 2 \mathrm{~b}(17 \%)$ and $1 / 2 \mathrm{c}(2.2 \%)$. 32 sequencetype (ST) were identified, of which 4 were new, and the predominant ones were ST1 (28.7\%) and ST2 (13.8\%). All the strains of L. monocytogenes were grouped in Lineages I and II. Conclusions: A great genetic variability was observed in the strains of $L$. monocytogenes analyzed, being predominant the ST1 and ST2, both belonging to Lineage I. Our results contribute to know the population structure of this pathogen in Chile and its presence in clinical samples, food and the environment.

Keywords: Listeria monocytogenes; serotyping; serotype; PFGE; MLST; lineage.

Palabras clave: Listeria monocytogenes; serotipificación; serotipo; PFGE; MLST; linaje.

\section{Introducción}

$L$ isteria monocytogenes es una bacteria grampositiva que se encuentra en el suelo, la vegetación y otras fuentes ambientales ${ }^{1}$. Este patógeno causa la listeriosis, que puede presentarse como gastroenteritis febril o en una forma invasora. La listeriosis invasora se manifiesta como meningitis, sepsis y aborto, y se asocia a una alta tasa de mortalidad en humanos y animales ${ }^{1}$. El tiempo de incubación de la listeriosis se estima de 2 a 88 días $^{2}$. Los pacientes inmunocomprometidos, los ancianos, las mujeres embarazadas y los neonatos corren un mayor riesgo de desarrollar listeriosis invasora $^{2}$. Debido a sus altas tasas de mortalidad y hospitalización (aproximadamente 25 y $97 \%$ de los pacientes, respectivamente), la caracterización de los aislados es esencial para identificar posibles brotes ${ }^{2}$. Listeria monocytogenes es una especie genéticamente diversa y ha sido caracterizada usando una variedad de técnicas de sub-tipificación para examinar su estructura poblacional y epidemiología. La serotipificación es un método clásico con un poder discriminatorio limitado ${ }^{3}$.
Este patógeno puede ser clasificado en 13 serotipos. De ellos, los serotipos 1/2a, 1/2b, 1/2c y 4 b causan la mayoría de los casos de listeriosis y, de éstos, el $4 \mathrm{~b}$ se asocia a la mayoría de los brotes de esta enfermedad ${ }^{3}$. La electroforesis en gel de campo de pulsado (PFGE, por sus siglas en inglés), hasta la fecha, ha sido el método con mayor discriminación y reproducibilidad para la subtipificación molecular de este patógeno y es considerada como el estándar de oro ${ }^{4,5}$. La tipificación de secuencias multilocus (MLST, por sus siglas en inglés), basada en la secuencia de nucleótidos de genes housekeeping, se usa para asignar secuencias tipo (ST) y grupos de variantes de locus único denominadas complejos clonales (CC). Se ha demostrado que para L. monocytogenes este método es altamente discriminatorio ${ }^{2,5}$. La técnica de MLST ha sido utilizada para investigar la amplia estructura poblacional de L. monocytogenes y sus resultados han confirmado la existencia de cuatro linajes genéticos discretos (I, II, III y IV), cada uno de los cuales puede causar enfermedad en humanos. Los resultados iniciales han indicado que los aislados de origen humano a menudo son miembros del Linaje I, mientras que los aislados ambientales, agrí-
'Subdepto. Genética Molecular, Instituto de Salud Pública de Chile.

${ }^{2}$ Subdepto. Enfermedades Infecciosas, Sección Bacteriología, Instituto de Salud Pública de Chile.

Los autores declaran ausencia de conflictos de interés.

Recibido: 20 de junio de 2018 Aceptado: 22 de julio de 2019

Correspondencia a: Soledad Ulloa Urrutia sole.ulloa@gmail.com 
colas y alimenticios se encontrarían preferentemente en el Linaje II $^{6}$. Sin embargo, los serotipos específicos del Linaje II (1/2a y $1 / 2 \mathrm{c})$ han sido aislados con la misma frecuencia que los serotipos del Linaje I (1/2b, 3b y 4b) de la listeriosis invasora en los países europeos. Los Linajes III y IV han sido aislados en raras ocasiones y aún no se han investigado en detalle ${ }^{6}$. Según Bundrant y cols. $2011^{7}$, y Den Bakker y cols., $2012^{8}$, los Linajes I y II están compuestos por los siguientes complejos clonales: (i) Linaje I CC1, CC2, CC3, CC5, CC6, CC59, CC217, CC363 y CC426 y (ii) Linaje II CC7, CC8, CC9, CC14, CC19, CC31, CC37, CC121, CC177, CC193 у CC204.

Múltiples brotes de L. monocytogenes ocurridos en diferentes países a causa de ciertos subtipos de PFGE se han denominado clones epidémicos (en inglés EC) ${ }^{9}$; sin embargo, es difícil evaluar su relación en ausencia de datos adicionales ${ }^{6}$. Por lo tanto, las relaciones genéticas más ampliamente estudiadas se han establecido mediante el uso de MLST. Incluso, hay reportes que muestran que los EC descritos inicialmente pertenecen a un CC o ST aislado y, en cambio, muchos de los CC descritos están distribuidos globalmente ${ }^{6}$. Los CC que corresponden a los siete EC son: CC1: ECI, CC2: ECIV, CC5: ECVI, CC6: ECII, CC7: ECVII, CC8: ECV y CC11: ECIII' ${ }^{6}$.

El Instituto de Salud Pública de Chile (ISP), realiza la vigilancia molecular de este patógeno a través de la técnica de PFGE, a partir de muestras de alimentos, clínicas (humanas) y ambientales. Mediante la vigilancia de este patógeno, en el año 2008 fue declarado el primer brote masivo con 78 casos pertenecientes a un mismo clon de L. monocytgenes, asociados al consumo de queso Brie y Camembert. Un segundo brote ocurrió en 2009 , el que fue detectado y relacionado con el consumo de salchichas contaminadas con un total de 10 casos reportados y relacionados a un mismo clon de L. monocytogenes ${ }^{10}$.

El objetivo principal de este estudio fue caracterizar genéticamente cepas de L. monocytogenes identificadas en Chile en el período 2007-2014.

\section{Materiales y Métodos}

\section{Selección de cepas desde base de datos de vigilancia por PFGE}

Se seleccionaron 94 cepas de L. monocytogenes de un total de 3.714 cepas presentes en nuestra base de datos de vigilancia por PFGE, las que representan 94 patrones diferentes de PFGE (pulsotipos), de un total de 225 pulsotipos presentes en esta base de datos. Estos patrones fueron seleccionados ya que estaban representados en tres o más cepas. Las cepas fueron recibidas por el laboratorio de referencia del ISP entre los años 2007 y 2014, desde
12 regiones del país y su origen es: $70(74,5 \%)$ de casos clínicos, $22(23,4 \%)$ de alimentos y $2(2,1 \%)$ de fuentes ambientales. La preparación de cada muestra recibida para vigilancia por PFGE y su posterior análisis, se realizó de acuerdo al protocolo estandarizado de la Red PulseNet (Centers for Disease Control and Prevention, CDC) (http://cdc.gov/pulsenet/pathogens/index.html). Brevemente, se realizó lisis celular a una suspensión bacteriana de L. monocytogenes y se utilizó agarosa $1 \%$ más dodecil sulfato de sodio (SDS) en solución $1 \%$ para la preparación de moldes de agarosa. El ADN se digirió con ApaI (40U/molde; Thermo Scientific). Salmonella serovar Braenderup cepa H9812 (ATCC BAA 664) se digirió con $\mathrm{XbaI}$ (50 U/molde; Thermo Scientific) y se usó como estándar de tamaño. La electroforesis se llevó a cabo a $14{ }^{\circ} \mathrm{C}$ durante $18 \mathrm{~h}$ a $6 \mathrm{~V} / \mathrm{cm}$ bajo rampa lineal con tiempos de 4 a $40 \mathrm{~s}$ y ángulo incluido de $120^{\circ}$. Los geles se tiñeron con bromuro de etidio y se visualizaron en un equipo GelDoc XR (BioRad). El análisis de los datos se realizó utilizando BioNumerics v6.6 (Applied Maths). Para ApaI, los diferentes patrones se agruparon en pulsotipos cuando fueron indistinguibles entre sí. El análisis de agrupamiento de patrones se realizó con el método UPGMA. Los parámetros de tolerancia y optimización se configuraron en $1,5 \%$ cada uno ${ }^{11}$.

\section{Serotipificación}

Las 94 cepas de L. monocytogenes seleccionadas fueron serotipificadas por reacción de polimerasa en cadena (RPC). Brevemente, se dejaron crecer cultivos durante la noche en agar infusión cerebro-corazón BHI (BioMérieux, Francia) a $37^{\circ} \mathrm{C}$. Se prepararon lisados bacterianos utilizando $94 \mu \mathrm{L}$ de una suspensión bacteriana en agua y $6 \mu \mathrm{L}$ de lisozima $20 \mathrm{mg} / \mathrm{mL}$ (Sigma-Aldrich, USA) y se incubó durante dos horas a $37^{\circ} \mathrm{C}$. Se centrifugó a 6.000 g por 2 min y se utilizó el sobrenadante como templado para la RPC múltiple, según lo descrito ${ }^{12,13}$. Se utilizó 5 $\mu \mathrm{L}$ de templado, partidores a una concentración final de $1 \mu \mathrm{M}$, GoTaq Green Master Mix (Promega) y agua libre de nucleasas hasta completar $50 \mu \mathrm{L}$.

\section{Tipificación de secuencias multilocus (MLST) y análisis filogenético}

Se realizó MLST en las 94 cepas como se describió anteriormente, mediante la amplificación y análisis de las secuencias de los 7 loci: abcZ, bglA, cat, dapE, dat, $l d h$ y $l h k A^{14}$. Se incorporaron nuevos alelos y perfiles en la base de datos internacional de MLST en http://bigsdb. pasteur.fr/listeria/.

Para cada cepa, la combinación de alelos en los siete loci define un perfil alélico o secuencia tipo (ST). El análisis del árbol de expansión mínima (en inglés: Minimum Spanning Tree-MST) se utilizó para inferir las relaciones entre los aislados y se realizó utilizando BioNumerics v6.6 
(Applied Maths). Un complejo clonal (CC) se definió en base al algoritmo eBURST ${ }^{15}$ con miembros ST que difirieron sólo en uno de los siete loci MLST, y el código numérico fue dado por http://bigsdb.pasteur.fr/listeria/. A partir de los resultados de MLST, se clasificó cada cepa en los Linajes descritos I, II, III y IV ${ }^{6}$.

\section{Resultados}

Para estudiar la diversidad genética de L. monocytogenes, se seleccionaron 94 cepas con base en sus patrones diferentes (pulsotipos) desde nuestra base de datos de vigilancia obtenida por PFGE, de un total de 225 pulsotipos diferentes. Los orígenes de estas cepas fueron: 70 cepas clínicas, 22 cepas de alimentos y 2 cepas ambientales. $\mathrm{Al}$ analizar el origen de los 94 pulsotipos en la base de datos completa; 50 se han encontrado exclusivamente en cepas aisladas de casos clínicos, 12 sólo en cepas aisladas desde alimentos, 30 en cepas aisladas desde casos clínicos y de alimento y 2 cepas en aislados desde casos clínicos, alimentarios y ambientales. Se construyó un dendrograma (UPGMA) basado en la presencia o ausencia de bandas en los patrones de PFGE. Los pulsotipos fueron divididos en tres grupos (pulsogrupos), según el porcentaje de similitud obtenido en el dendrograma: los pulsogrupos A y B presentan $80 \%$ de similitud entre sí, mientras el pulsogrupo $\mathrm{C}$ presenta $76 \%$ de similitud con A y B (Figura 1). En el dendrograma obtenido por PFGE, el pulsogrupo A, compuesto por 25 pulsotipos, agrupó 18 cepas del serotipo 4 b y 7 del serotipo $1 / 2$ a. El pulsogrupo $\mathrm{B}$, compuesto por 51 pulsotipos agrupó 34,16 y 1 cepas de los serotipos $4 \mathrm{~b}, 1 / 2 \mathrm{~b}$ y $1 / 2 \mathrm{a}$, respectivamente; el pulsogrupo $\mathrm{C}$, compuesto por 18 pulsotipos, agrupó 15 y 3 cepas de los serotipos $1 / 2$ a y $1 / 2$ c, respectivamente (Figura 1). Los pulsotipos de origen clínico, alimentos y el medio ambiente se distribuyeron en forma aleatoria entre los tres pulsogrupos.

\section{Serotipificación de cepas de L. monocytogenes mediante RPC}

Las cepas se agruparon en 4 de los 13 serotipos descritos: 1/2a, 1/2b, 1/2c y el complejo 4b-4d-4e. La RPC múltiple utilizada no distingue entre los serotipos del complejo $4 \mathrm{~b}-4 \mathrm{~d}-4 \mathrm{e}^{7}$. Sin embargo, como los serotipos $4 \mathrm{~d}$ y $4 \mathrm{e}$ no se han encontrado en muestras humanas y de alimento $^{16}$, las cepas pertenecientes al complejo $4 \mathrm{~b}-4 \mathrm{~d}-4 \mathrm{e}$ de nuestro estudio fueron consideradas serotipo $4 \mathrm{~b}$. El serotipo más común fue el 4b (52 cepas, 55,3\%), seguido por $1 / 2 \mathrm{a}, 1 / 2 \mathrm{~b}$ y $1 / 2 \mathrm{c}$ con frecuencias del $24,5 \%$ (23 cepas), $17,0 \%$ ( 16 cepas) y $3,2 \%$ ( 3 cepas), respectivamente. Los serotipos de cepas de origen clínico, alimentos y el medio ambiente se distribuyeron en forma aleatoria entre los tres pulsogrupos.

\section{Tipificación de secuencias multilocus}

En todas las cepas se amplificaron los 7 loci y el análisis de los alelos permitió identificar 32 STs diferentes, 28 de las cuales ya habían sido descritas en la base de datos internacional (http://bigsdb.pasteur. fr/listeria/) y 4 STs nuevas: ST734, ST735, ST736 y ST737. La mayoría de las $\mathrm{STs}^{30}$ se agruparon en 25 complejos clonales y sólo dos ST no fueron agrupables. Las ST predominantes, identificadas en $50 \%$ de las cepas estudiadas, correspondieron a las ST1 $(28,7 \%)$, ST2 $(13,8 \%)$ y ST6 $(7,4 \%)$, cuyos pulsotipos fueron serotipo $4 \mathrm{~b}$, a excepción de una cepa que resultó serotipo 1/2a. La ST1, identificada en 27 pulsotipos, sólo se encontró en pulsotipos cuyas cepas eran de origen clínico o clínico y alimentario, pero no de origen alimentario o ambiental, exclusivamente. Las ST detectadas solamente en pulsotipos con cepas de origen clínico fueron: ST36, ST101, ST177, ST193, ST279, ST388, ST735 y ST737. La ST204 fue detectada únicamente en un pulsotipo con cepas de origen alimentario. Sólo dos pulsotipos comparten muestras de origen clínico, alimentario y ambiental de forma simultánea. Ambos pertenecen al pulsogrupo B y en la Figura 1 corresponden a Origen PT: C + AL + AM, cuyas STs son 426 y 6 . Estas STs fueron identificadas también en otros pulsotipos de origen exclusivo clínico o alimentario. De las 94 cepas estudiadas, 68 se agruparon en el Linaje I: CC1 $(n=29)$, CC2 ( $n=13), \operatorname{CC} 3(n=4), \operatorname{CC} 5(n=3), \operatorname{CC} 6(n=7)$, CC59 ( $=1), \operatorname{CC} 217(n=1), \operatorname{CC} 363(n=2), \operatorname{CC} 388$ $(n=1), \operatorname{CC} 392(n=2), \operatorname{CC} 426(n=3)$ y 2 cepas $\sin$ CC; y 26 en el Linaje II: CC7 ( $n=1)$, CC8 $(n=3)$, CC9 $(n=3), \operatorname{CC} 14(n=2), \operatorname{CC} 19(n=1), \operatorname{CC} 31(n=1), \operatorname{CC} 36$ $(\mathrm{n}=2), \operatorname{CC} 37(\mathrm{n}=3), \operatorname{CC} 101(\mathrm{n}=1), \operatorname{CC} 121(\mathrm{n}=5)$, CC124 (n=1), CC177 (n=1), CC193 (n=1) y CC204 $(n=1)$. No se identificaron cepas pertenecientes a los Linajes III y IV (Figura 1).

\section{Discusión}

El ISP de Chile realiza la vigilancia molecular de $L$. monocytogenes por PFGE desde el año 2007. Durante este tiempo, se ha creado una base de datos con 3.714 cepas, que incluye 924 cepas obtenidas de casos clínicos, 2.778 cepas obtenidas de alimentos y 12 cepas obtenidas del ambiente. En esta vigilancia se han identificado 225 pulsotipos durante el período estudiado. En este estudio, se seleccionaron 94 pulsotipos representativos de dicha base de datos y se estudiaron mediante serotipificación y MLST. El análisis mediante UPGMA permitió la identificación de tres pulsogrupos A, B y C, compuestos por 25,51 y 18 pulsotipos, respectivamente.

El serotipo predominante fue el $4 \mathrm{~b}$, seguido de $1 / 2 \mathrm{a}$, $1 / 2$ b y $1 / 2$ c. Esta misma distribución fue encontrada por 


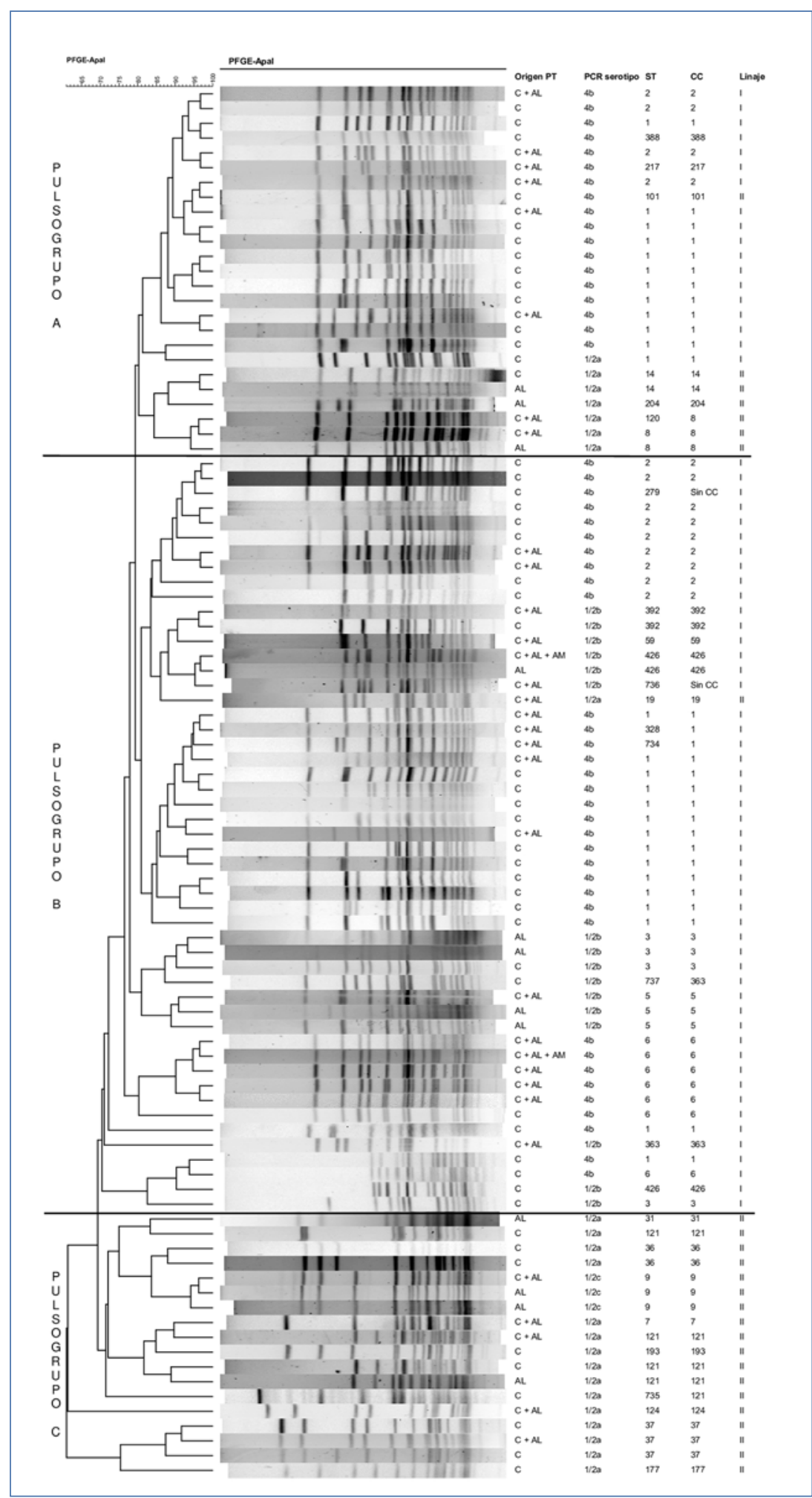

Figura 1. Relación entre pulsotipos basada en PFGE. 94 cepas de L. monocytogenes se analizaron por PFGE usando Apal y el dendrograma de los pulsotipos se construyó basado en el método UPGMA. Los correspondientes: origen de pulsotipo (PT), RPC serotipo, ST, CC y linaje se muestran a la derecha del dendrograma.
Montero y cols. $2015^{17}$, en muestras de alimentos chilenas y por Toledo y cols. $2018^{18}$, en muestras clínicas y no clínicas chilenas.

En $99 \%$ de los casos de este estudio, diferentes pulsotipos que compartieron el mismo ST también tuvieron el mismo serotipo; a excepción de un pulsotipo del pulsogrupo A con ST1, cuyo serotipo es 1/2a y los otros 25 pulsotipos con ST1 (presentes en pulsogrupos A y B) tienen serotipo $4 \mathrm{~b}$. Cepas de un mismo ST y con diferentes serotipos ya fueron descritas antes por Yin y cols. $2015^{19}$, quienes sugieren que L. monocytogenes evoluciona y genera diferentes serotipos como una manera de adaptarse a diferentes nichos ecológicos. De igual manera, Cordero y Polz, 2014 ${ }^{20}$, proponen que la variación del genoma de L. monocytogenes estaría asociada a interacciones sociales y ecológicas. En este estudio, esto ocurrió sólo en una cepa de origen clínico.

Las cepas del serotipo $4 \mathrm{~b}$ se agruparon solamente en los pulsogrupos A y B (Figura 1). Por otra parte, 83,3\% de las cepas del pulsogrupo $\mathrm{C}$ pertenecieron al serotipo $1 / 2$ a y $100 \%$ de las cepas del grupo $C$ pertenecieron al Linaje II. Los 11 CC identificados por Toledo y cols. $2018^{18}$, en muestras chilenas fueron también encontrados en las muestras incluidas en este trabajo. Los CC más comunes dentro de los Linajes I y II fueron CC1 $(30,8 \%)$ y CC121 (5,3\%), respectivamente. Universalmente, se han descrito siete clones epidémicos, los que están asociados a diferentes complejos clonales ${ }^{6}$. En este estudio se identificaron seis de los siete clones epidémicos en 56 de las cepas analizadas. En otras 38 cepas se identificaron STs o CCs que no estaban relacionadas con los clones epidémicos previamente descritos. Los clones epidémicos ECI y ECIV, correspondientes a los $\mathrm{CC} 1$ y $\mathrm{CC} 2$, respectivamente, fueron predominantes con 51,8 y $23,2 \%$ de los pulsotipos que fue posible asociar a clones epidémicos en este trabajo. Estos clones epidémicos han sido reportados como responsables de brotes en diferentes países: Suiza (1983 a 1987), E.U.A. (Boston 1979, Massachusetts 1983 y California 1985) ${ }^{21}$, Nueva Escocia (1981), Dinamarca (1985 a 1987), Reino Unido (1987), Francia (1992) y E.U.A. (1998, 1999 y 2000)22. Una muestra clínica se agrupó con ST101, CC101. En 2014, Haase y cols. ${ }^{6}$, describieron al CC101 como predominante en muestras humanas; sin embargo, probablemente no fue descrito como un clon epidémico, debido a que desde 1950s ha sido reportado infrecuentemente, a excepción de un brote en Italia durante 2006 y $2010^{23}$. En este estudio no se identificaron cepas pertenecientes al ECIII.

En relación a los linajes descritos para $L$. monocytogenes, 68 cepas se agruparon en el Linaje I y 26 en el Linaje II. En las 94 cepas se identificaron los 9 y 11 complejos clonales descritos para los Linajes I y II, respectivamente. El pulsotipo C contiene 15 cepas del serotipo 1/2a (de un 
total de 18). El 77,8\% de este pulsogrupo está compuesto por cepas del Linaje II. Similares resultados han sido obtenidos entre el serotipo $1 / 2$ a y el Linaje II en otros países $^{24,25}$.

Cuando se analizó mediante el método UPGMA la relación clonal entre los pulsotipos de L. monocytogenes, se observó que aquellos obtenidos de cepas aisladas de casos clínicos se distribuyeron con la misma frecuencia en los tres pulsogrupos encontrados. Sin embargo, los pulsotipos encontrados únicamente en cepas provenientes de alimentos, se agruparon con mayor frecuencia en el pulsogrupo C, el que está compuesto, en $100 \%$, por cepas pertenecientes al Linaje II, lo que se correlaciona con lo descrito para este linaje, donde predominan las muestras de alimentos ${ }^{25}$. No obstante, esta diferencia no es estadísticamente significativa $(p<0,05)$.

\section{Conclusiones}

Los resultados obtenidos en este trabajo permitieron caracterizar genéticamente cepas de L. monocytogenes obtenidas en Chile durante el período 2007-2014, mediante estudio de serotipificación y MLST. Se observó una gran variabilidad genética en las cepas analizadas, siendo predominantes las secuencias tipo ST1 y ST2, ambas pertenecientes al Linaje I. Nuestros resultados contribuyen a conocer la estructura poblacional de este patógeno en Chile y su presencia en diferentes tipos de muestras.

La vigilancia epidemiológica que se realiza en forma permanente en nuestro país, ha demostrado ser fundamental para conocer la diversidad genética y distribución de pulsotipos en muestras clínicas, alimentos, y aislamientos ambientales para prevenir nuevos brotes epidémicos.
Agradecimientos. A Daniela Torrejón y María Sandoval por su excelente labor técnica en el desarrollo de este trabajo.

\section{Resumen}

Introducción: Listeria monocytogenes es un patógeno transmitido por alimentos que causa listeriosis, una enfermedad que puede presentarse como gastroenteritis febril o en una forma invasora que tiene altas tasas de mortalidad. Hasta el momento, ha sido poco estudiada la diversidad genética de cepas de L. monocytogenes aisladas desde pacientes, alimentos y fuentes ambientales en Chile. Objetivo: Caracterizar genéticamente cepas de L. monocytogenes de estos tres orígenes recibidas por el Instituto de Salud Pública de Chile (ISP) entre los años 2007 y 2014. Material y Métodos: Se seleccionaron 94 cepas de L. monocytogenes correspondientes a 94 pulsotipos diferentes identificados por electroforesis en gel de campo pulsado (PFGE), se extrajo ADN y se realizó serotipificación mediante reacción de polimerasa en cadena (RPC) y tipificación de secuencias multilocus (MLST). Resultados: El serotipo más común fue $4 \mathrm{~b}$ (55,3\%), seguido de $1 / 2 \mathrm{a}(25,5 \%), 1 / 2 \mathrm{~b}(17 \%)$ y $1 / 2 \mathrm{c}$ $(2,2 \%)$. Se identificaron 32 secuencias tipo (ST), de las cuales cuatro fueron nuevas, y las predominantes fueron ST1 $(28,7 \%)$ y ST2 $(13,8 \%)$. La totalidad de las cepas se agrupó en los Linajes I y II. Conclusiones: Se observó una gran variabilidad genética en las cepas de L. monocytogenes analizadas, siendo predominantes las secuencias tipo ST1 y ST2, ambas pertenecientes al Linaje I. Nuestros resultados contribuyen a conocer la estructura poblacional de este patógeno en Chile y su presencia en muestras clínicas, alimentos y el medio ambiente.

\section{Referencias bibliográficas}

1.- $\quad$ Farber J M, Peterkin P I. Listeria monocytogenes, a food-borne pathogen. Microbiol Rev 1991; 55: 476-511. PMID: 1943998.

2.- Yde M, Naranjo M, Mattheus W, Stragier P, Pochet B, Beulens K, et al. Usefulness of the European epidemic intelligence information system in the management of an outbreak of listeriosis, Belgium, 2011. Eurosurveill 2012; 17(38). http://www.eurosurveillance.org/ ViewArticle.aspx?ArticleId=20279.

3.- Nightingale K, Bovell L, Grajczyk A, Wiedmann M. Combined $\operatorname{sig} B$ allelic typing and multiplex PCR provide improve discriminatory power and reliability for Listeria monocytogenes molecular serotyping. J Microbial Biotech 2007; 68: 52-9. doi: 10.1016/j.mimet.2006.06.005.
4.- Gerner-Smidt P, Hise K, Kincaid J, Hunter S, Rolando S, Hyytiä-Trees E, et al. PulseNet USA: a five-year update. Foodborne Pathog 2006; 3 (1): 9-19. doi: 10.1089/fpd.2006.3.9.

5.- Chenal-Francisque V, Diancourt L, Cantinelli T, Passet V, Tran-Hykes C, Bracg-Dieve $\mathrm{H}$, et al. Optimized multilocus variablenumber tandem-repeat analysis assay and its complementarity with pulsed-field gel electrophoresis and multilocus sequence typing for Listeria monocytogenes clone identification and surveillance. J Clin Microbiol 2013; 51 (6): 1868-80. doi: 10.1128/JCM.00606-13.

6.- Haase J, Didelot X, Lecuit M, Korkeala H. L. monocytogenes MLST Study Group, Achtman M. The ubiquitous nature of Listeria monocytogenes clones: a large-scale Multilocus Sequence Typing study. Environ Microbiol 2014; 16 (2): 405-16. doi: 10.1111/14622920.12342 .
7.- $\quad$ Bundrant B, Hutchins T, Den Bakker $\mathrm{H}$, Fortes E, Wiedmann M. Listeriosis outbreak in dairy cattle caused by an unusual Listeria monocytogenes serotype $4 \mathrm{~b}$ strain. J Vet Diagn Invest 2011; 23: 155-8. doi: 10.1177/104063871102300130.

8.- Den Bakker H C, Desjardins C, Griggs A, Peters J, Zeng Q, Young S, et al. Evolutionary dynamics of the accesory genome of Listeria monocytogenes. PLoS One 2013; 8 (6): e67511. doi: 10.1371/journal.pone.0067511

9.- Cantinelli T, Chenal-Francisque V, Diancourt L, Frezal L, Leclercq A, Wirth T, et al. "Epidemic clones" of Listeria monocytogenes are widespread and ancient clonal groups. J Clin Microbiol 2013; 52 (11): 3770-9. doi: 10.1128/ JCM.01874-13

10.- MINSAL. Informe de Listeria, Chile 2010. Disponible en http://epi.minsal.cl/vigilanciaepidemiologica/enfermedades-de-notificacion- 
obligatoria/listeria (Acceso el 27 de abril de 2018).

11.- Hunter S, Vauterin P, Lambert-Fair M A, Van Duyne M S, Kubota K, Graves L, et al. Establishment of a universal size standard strain for use with the PulseNet standardized pulsefield gel electrophoresis protocols: converting the national databases to the new size standard. J Clin Microbiol 2005; 43(3): 1045-50. doi: 10.1128/JCM.43.3.1045-1050.2005.

12.- Doumith M, Buchrieser C, Glaser P, Jacquet Ch, Paul M. Differentiation of major Listeria monocytogenes serovars by multiplex PCR. J Clin Microbiol 2004; 3819-22. doi: 10.1128/ JCM.42.8.3819-3822.2004.

13.- Burall L S, Simpson A C, Datta A R. Evaluation of a serotyping scheme using a combination of an antibody-based serogrouping method and a multiplex PCR assay for identifying the major serotypes of Listeria monocytogenes. J Food Prot 2011; 74: 403-9. doi: 10.4315/0362-028X. JFP-10-355.

14.- Ragon $M$, Wirth $T$, Hollandt $F$, Lavenir R, Lecuit M, Le Monnier A, et al. A new perspective on Listeria monocytogenes evolution. PLoS Pathog 2008; e1000146. doi: 10.1371/jounal.ppat.1000146.

15.- Feil E, Li BC, Aanensen D, Hanage W, Spratt B. eBURST: Inferring patterns of evolutionary descent among clusters of related bacterial genotypes from multilocus sequence typing data. J Bacteriol 2005; 186 (5): 1518-30. doi: 10.1128/JB.186.5.15181530.2004

16.- Braga V, Vásquez S, Vico V, Pastorino V, Mota M, Legnani M, et al. Prevalence and serotype distribution of Listeria monocytogenes isolated from foods in Montevideo-Uruguay. Braz J Microbiol 2017; 48: 689-94. doi: 10.1016/j. bjm.2017.01.010.

17.- Montero D, Bodero M, Riveros G, Lapierre L, Gaggero A, Vidal R, et al. Molecular epidemiology and genetic diversity of Listeria monocytogenes isolates from a wide variety of ready-to-eat foods and their relationship to clinical strains from listeriosis outbreaks in Chile. Front Microbiol 2015; 6: 1-8. 384. doi: 10.3389/fmicb.2015.00384

18.- Toledo V, den Bakker H C, Hormazábal J C, González-Rocha G, Bello-Toledo H, Toro M, et al. Genomic diversity of Listeria monocytogenes isolated from clinical and nonclinical samples in Chile. Genes 2018; 9(8): pii E396. doi: 10.3390/genes9080396

19.- Yin Y, Tan W, Wang G, Kong S, Zhou X, Zhao D, et al. Geographical and longitudinal analysis of Listeria monocytogenes genetic diversity reveals its correlation with virulence and unique evolution. Microbiol Res 2015; 175: 84-92. doi: 10.1016/j.micres.2015.04.002.

20.- Cordero O, Polz M. Explaining microbial genomic diversity in light of evolutionary ecology. Nat Rev Microbiol 2014; 12: 263-73. doi: $10.1038 /$ nrmicro3218

21.- Piffaretti J C, Kressebuch H, Aeschbacher M, Bille J, Bannerman E, Musser J M. Genetic characterization of clones of the bacterium Listeria monocytogenes causing epidemic disease. Proc Natl Acad Sci USA 1989; 86: 3818-22. doi: 10.1073/pnas.86.10.3818.

22.- Kathariou S. Listeria monocytogenes virulence and pathogenicity, a food safety perspective. J Food Prot 2002; 65: 1811-29. https://doi. org/10.4315/0362-028x-65.11.1811.

23.- Mammina C, Parisi A, Guaita A, Aleo A, Bonura C, Nastasi A, et al. Enhanced surveillance of invasive listeriosis in the Lombardy region, Italy, in the years 20062010 reveals major clones and an increase in serotype 1/2a. BMC Infect Dis 2013; 13: 152. doi: 10.1186/1471-2334-13-152.

24.- Zhang C, Zhang M, Ju J, Nietfeldt J, Wise $\mathrm{J}$, Terry $\mathrm{P}$, et al. Genome diversification in phylogenetic Lineages I and II of Listeria monocytogenes: identification of segments unique to Lineage II in populations. J Bacteriol 2003; 185 (18): 5573-84. doi: 10.1128/ JB.185.18.5573-5584.2003.

25.- Wang Y, Zhao A, Zhu R, Lan R, Jin D, Cui Z, et al. Genetic diversity and molecular typing of Listeria monocytogenes in China. BMC Microbiol 2012; 12 (119). doi: 10.1186/14712180-12-119. 